\title{
High resolution Imaging of possible microquasars
}

\section{Pandey ${ }^{*}$}

CNRS FRE 2591/CEA Saclay, DSM/DAPNIA/SAP, F-91191 Gif sur Yvette Cedex, France

E-mail: mamtapan@gmail.com

\section{Z. Paragi}

JIVE, MTA Research Group for Physical Geodesy and Geodynamics, Dwingeloo, Netherlands

E-mail: zparagi@jive.nl

\section{P. Durouchoux}

ESIEA-DRDI, 72 avenue Maurice Thorez, 94200, Ivry-sur-Seine, France

E-mail: pdurouchoux@hotmail.com

\section{H. Bignall}

JIVE, Dwingeloo, Netherlands

E-mail: bignallejive.nl

We report high resolution e-VLBI observations on two possible microquasars IGR J17303-0601 and IGR J18406-0539 with the European VLBI Network (EVN) at $5 \mathrm{GHz}$ in December 2006 and September 2007. These sources had candidate optical counterparts showing binary nature, and had radio counterparts in NVSS $(1.4 \mathrm{GHz})$ or GMRT $(0.610 \mathrm{GHz}$ and $1.28 \mathrm{GHz})$ observations within the INTEGRAL position errors. Our goal was to detect emission on millarcsecond scales to see whether they have associated compact core or core-jet structures as most microquasars do. We also wanted to verify, if the proposed NVSS/GMRT possible radio counter parts satisfy such morphology. e-VLBI observations allow us to achieve milliarcsec scale resolution and explore the detailed morphology of such sources. Radio maps of high quality can be quickly (within hours of observations) produced with the e-VLBI. 6 telescopes were involved for EVN observation at 5 $\mathrm{GHz}$ correlated in real time at the Joint Institute for VLBI in Europe (JIVE). We detect the NVSS radio source near IGR J17303-0601, which is marginally consistent with the ROSAT position of the source. However our accurate VLBI coordinates make it absolutely clear that the radio source is unrelated to the suggested optical counterpart of IGR J17303-0601. Thus, there is likely no association between the radio and the optical/X-ray sources. We do not detect the GMRT/NVSS source near IGR J18406-0539 on milliarcsecond scales. Our e-VLBI observations do not support the microquasar nature of these two INTEGRAL sources.

Bursts, Pulses and Flickering:Wide-field monitoring of the dynamic radio sky

June 12-15 2007

Kerastari, Tripolis, Greece

\footnotetext{
* Speaker.
}

$\dagger$ 


\section{Introduction}

A majority of bright Galactic X-ray sources are in binary systems with an accreting black hole or a neutron star as the compact companion. Apart from persistent X-ray emission from a number of these binaries, a large population of these systems are transient in nature, which brighten up on occasion and individually each source has characteristics of its own. Since the observation of super luminal outflow during the transient burst of GRS 1915+105 (Mirabel et al. 1994), a new sub class, characterised by jets in X-ray binaries, has come into being. The radio morphology of these sources suggests similarity with quasars but at a scaled down magnitude of the order of six hence are named microquasars. Bright Galactic X-ray sources, most of which are binary systems showing variable hard XRB-like spectra, have been discovered by the INTEGRAL, Gamma-Ray Satellite which performs Spectral analysis (20 keV - 8 MeV), Imaging (15 keV - $10 \mathrm{MeV}$ ) and monitoring of sources. There is increasing evidence that the hard X-ray emission produced by accretion onto stellar mass black holes is always associated with the production of relativistic jets (Fender et al. 1999). Several of the X-ray binaries have been found to emit radio emission, which can be highly variable in intensity and spatial structure. Since the number of these known microquasars is relatively small, any new discoveries would contribute greatly to our understanding of this class of objects.

We carried out short observations on two microquasar candidates, IGR J17303-0601 and IGR J18406-0539 at high resolution with the EVN, close in time to the INTEGRAL Galactic Plane survey during December 2006 and Sep 2007. INTEGRAL data on IGR J18406-0539 is only available for December 2006 and the source showed slight activity in the X-ray light curve, while emitting in hard X-ray regime. No data is available in the public archive for the other source and for September observations. Our goal was to establish their compact (or compact-jet) nature, and confirm the suitability of the selected phase-reference sources. Such exploratory observations are important for further study of these systems. We present the results of our observations in this paper. In Sect. 2 we present the source selection criteria and observation details. The results of our observations are discussed in sect. 3. Conclusion on this exploratory experiment are presented in sect. 4.

\section{Observation and Analysis}

\subsection{Source selection criteria}

We carried out a radio survey of INTEGRAL sources and known microquasars with the GMRT to explore their nature at very low energies. Forty INTEGRAL sources were observed at $0.610 \mathrm{GHz}$ and $1.28 \mathrm{GHz}$. Based on the radio morphology, these sources could be grouped into three different categories: diffuse sources, point sources and double sources. At GMRT resolution only four point sources were detected within the INTEGRAL error boxes that were likely Galactic in origin, and their spectral nature was similar to X-ray binaries. Three of them were radio transients (likely associated to IGR J17091-3624, IGR J17303-0601, and IGR J17464-3213), and one was persistent (likely associated with J18406-0539) (Pandey et. al. 2006a and b). The radio observations on these four sources with various observatories suggest they are variable; however, jet structure 
could not be confirmed. Two of the above mentioned sources were at high enough declination for the EVN: IGR J17303-0601, and J18406-0539

\subsection{EVN observations}

We requested short e-VLBI observations with the EVN on likely radio counterparts of both IGR J17303-0601 and IGR J18406-0539 at 5 GHz (in total 5 hours) in December 2006 and September 2007. Since these sources lie in a region where there is a high risk of scatter broadening, we preferred $5 \mathrm{GHz}$ over $1.67 \mathrm{GHz}$. The e-EVN array consisted of Cambridge, Jodrell Bank (MkII), Medicina, Torun and Westerbork (phased array); the data were correlated in real time at the Joint Institute for VLBI in Europe (JIVE). The resolution of $\sim 6 \times 10$ mas was suitable for our goals. The data was acquired at the rate of $256 \mathrm{Mbps}$, with $8 \mathrm{MHz}$ sub bands and 2-bit sampling, dual polarization, giving $4 \mathrm{~TB}$ of raw sampled data over the 5 hours of the whole experiment on 27th Dec, 2006 and a similar amount in 4 hours during the 6th September, 2007 experiment. The observations were made using the phase-reference technique with a cycle of $150 \mathrm{~s}$ on the target source (IGR J17303-0601 and IGR J18406-0539) and 80s on the phase reference source (J17280350 (200 mJy source 2.2 degrees away) and J1846-0651 (100 mJy source 1.8 degrees away)). For fringe-finding, we suggested the nearby compact source J1743-0350 (2-4 Jy). Additional calibrators were 3C138 and 3C286 for Westerbork synthesis array amplitude calibration. In September 2007 we requested a minimum 2 hours of observation time to check the compactness of the VLA counterpart of IGR J17303-0601. The observations settings were similar to those in Dec 2006.

The initial data reduction was performed using AIPS. The system temperature and gain calibration were applied and the AIPS task FRING was used to solve for the delay across the base bands and delay rate to give a better signal to noise ratio and correct for various atmospheric and instrumental errors. A self-calibrated image of the phase calibrator was produced using package DIFMAP, enabling further calibration using AIPS + DIFMAP to produce final images of the target sources.

\section{Results}

IGR J17303-0601: This source was detected in the Norma arm region (Walter et al. 2004) and in coincidence with the ROSAT source 1RXS J173021.5-055933 (Voges et al. 1999, Stephen et al. 2005). The ROSAT position coordinates are, RA: $17 \mathrm{~h} 30 \mathrm{~m} 21.50 \mathrm{~s}$ and DEC: $-05 \mathrm{~d} 59^{\prime} 33.5^{\prime \prime}$ (J 2000). Two optical objects with $R \sim 15.5$ and $R \sim 18$ are found within the ROSAT error box of $7^{\prime \prime}$ in the Digitized Sky Survey (DSS) field (Monet et al. 2003, Stephen et al. 2005). Two near-infrared sources in the (2-MASS survey) are also coincident with the optical sources. The optical spectra of both these sources acquired with the Bologna Astronomical Observatory show features identical to other X-ray emitting objects (Masetti et al. 2004). The brighter source within the ROSAT error box was considered to be the optical counterpart to IGR J17303-0601. All the optical emission lines of this object are at zero redshift, indicating its Galactic origin. The presence of the He II line strongly indicates that this object is undergoing mass accretion onto a compact star (e.g. van Paradijs \& McClintock 1995, Barlow et al. 2006), thereby suggesting the X-ray source to be a low mass X-ray binary. The optical photometry of the source, however suggests the hard X-ray source is an intermediate polar with a spin period of $128 \mathrm{~s}$ (Gansicke et al. 2005). 


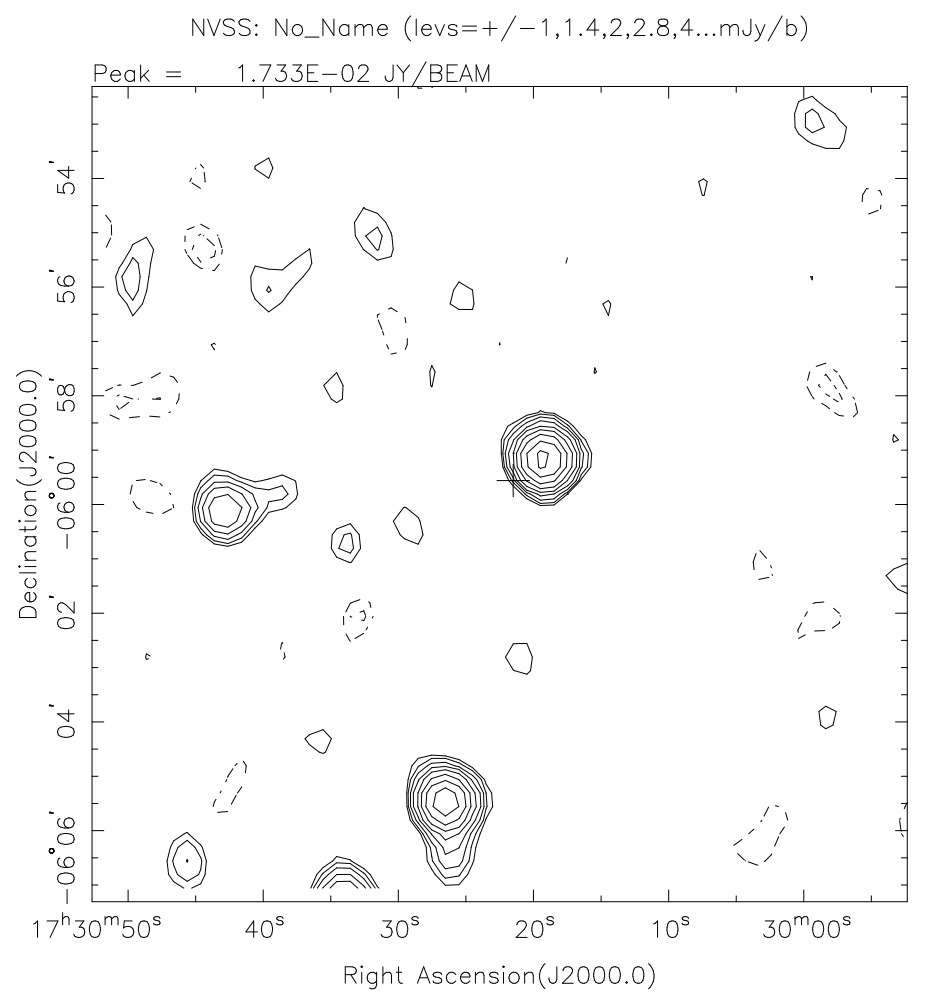

Figure 1: NVSS image of the region around IGR J17303-0601 at $1.4 \mathrm{GHz}$. The cross mark shows the ROSAT position of the source

The NVSS data at $1.4 \mathrm{GHz}$ showed a compact point source of flux density $\sim 18 \mathrm{mJy}$ at 1.4 $\mathrm{GHz}$ and marginally coincident (39" offset) with the INTEGRAL and ROSAT source position (see Fig 1.). The NVSS position was RA: $17 \mathrm{~h} 30 \mathrm{~m} 19.20$ s and DEC: $-05^{0} 59^{\prime} 10.5^{\prime \prime}$ (J 2000). Two other compact field sources with radio flux density $\sim 5 \mathrm{mJy}$ and RA: $17 \mathrm{~h} 30 \mathrm{~m} 45.48$ and DEC: $-06^{0} 01^{\prime} 57.17^{\prime \prime}$ and a radio flux density of $\sim 16$ mJy and RA: $17 \mathrm{~h} 30 \mathrm{~m} 28.41$ and DEC: $-06^{0} 05^{\prime}$ $56.27^{\prime \prime}$, were clearly detected within the field and lie outside the INTEGRAL error circle. No radio counterpart was detected with the GMRT suggesting either a synchrotron self absorption or nonpresence of low frequency emitting component, as seen in the case of other microquasars (Pandey et al. 2006b). It is also possible that the source was in high/soft X-ray state and hence the radio emission was quenched.

During e-VLBI observations with the EVN in December 2006, on IGR J17303-0601 the Xray position was considered in order to detect any radio emission within the ROSAT error circle. No radio emission was detected at the ROSAT position. The possible NVSS radio counterpart lies several arc seconds off, which means that during the observations this source was out of the field of view limited by the phased Westerbork array. The rms noise was $\sim 153$ microjansky/beam. We have also analysed the WSRT local interferometry data on the source and detected an unresolved source of $\sim 10$ mJy radio flux density at $5 \mathrm{GHz}$, in agreement with the NVSS source coordinates. The $\mathrm{rms}$ recorded in the map is $0.19 \mathrm{mJy} / \mathrm{beam}$.

During the September 2007 e-VLBI observation run, the nearby NVSS source was observed. A well resolved source of total flux density $\sim 10$ mJy was detected during this observation. Fig. 2 


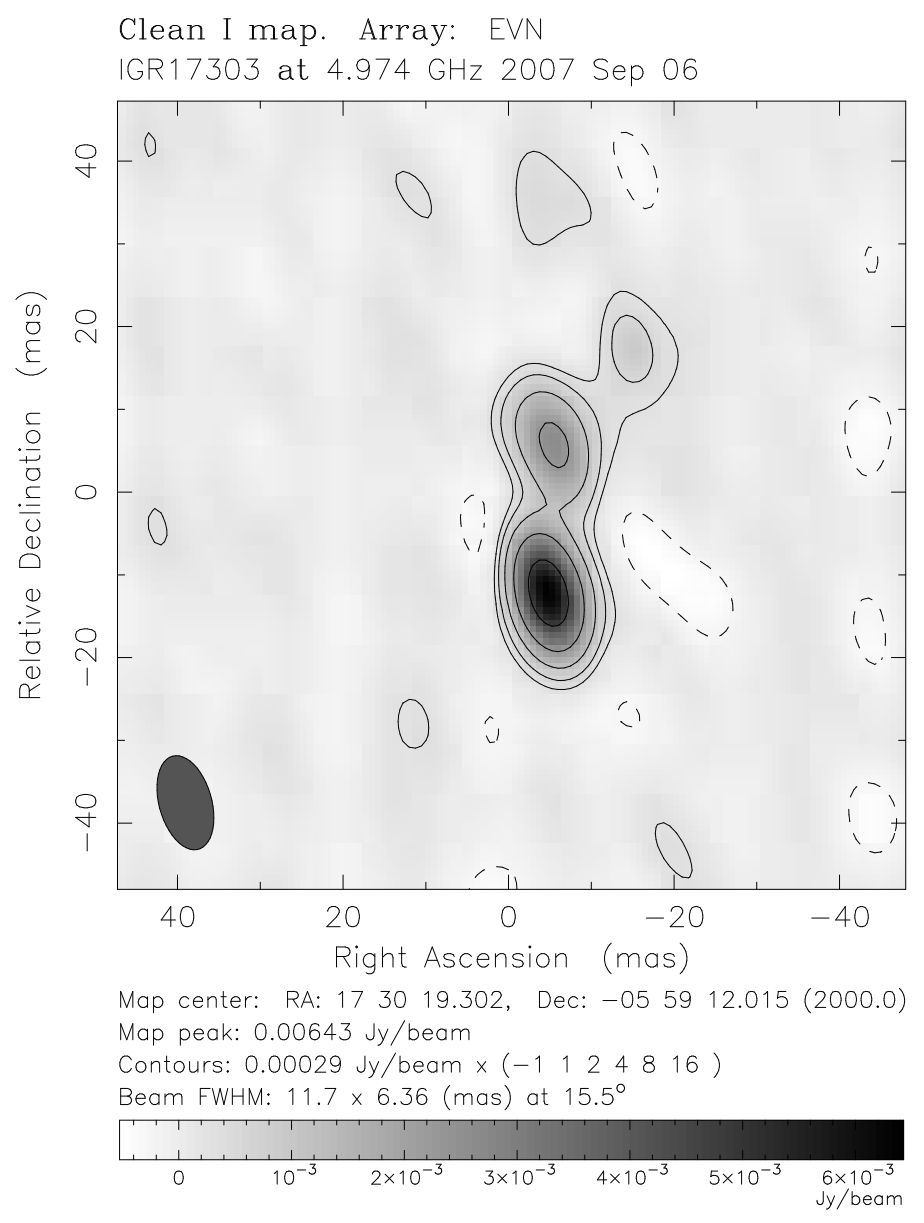

Figure 2: e-VLBI image of the NVSS source near IGR J17303-0601 at $5 \mathrm{GHz}$

shows the high resolution e-VLBI image of at $5 \mathrm{GHz}$. The source is well resolved and lies $\sim 2.11^{\prime \prime}$ away from the NVSS source position. This source was persistent during our observation above 1.4 $\mathrm{GHz}$ and has a spectral index of -0.46 between 1.4 and $5 \mathrm{GHz}$. To summarize, no radio emission was detected in the ROSAT error circle and the possible NVSS counterpart though being resolved at e-VLBI scales, showed no radio variability, and the measured VLBI position completely rules out the association with the optical source that is regarded as the counterpart to the X-ray source. This suggest that the NVSS radio source may be a possible background object and unassociated with the INTEGRAL source, IGR J17303-0601.

IGR J18406-0539: This source was discovered during the observations of the Sagittarius arm region by the IBIS telescope in 2003 (Belanger et al. 2004). We carried out cross identification of this source, with the data available from various catalogues, to identify the nature of the source. A hard X-ray source, AX J1840.4-0537 was discovered during ASCA observations with RA: 18h $40 \mathrm{~m} 24 \mathrm{~s}$ and DEC: $-05^{0} 37^{\prime} 00^{\prime \prime}$ lies within the position error circle of the source (Bamba et al. 2003). The field is further complicated by the presence of an optical source, GSC2.2 with magnitudes $B=16$ and $R=19$ and position RA: $18 \mathrm{~h} 40 \mathrm{~m}$ 38.094s and DEC: $-05^{0} 43^{\prime} 19.30^{\prime \prime}$ lying within error circle (Monet et al. 1998). Later, Masetti et al. (2006) confirmed the high mass X- 
ray binary nature of the source via optical observations. An IR point source, IRAS J18379-0546 (Cutri et al. 2003), with coordinates RA: $18 \mathrm{~h} 40 \mathrm{~m} 38.04 \mathrm{~s}$, DEC: $-05^{0} 43^{\prime} 20^{\prime \prime}$ and magnitudes $J=12.89, H=11.91, K=11.61$, also lies within the $2 \sigma$ position error ellipse of the hard X-ray source.

During GMRT observations, a point source was detected with a radio flux density of $\sim 28 \mathrm{mJy}$ and at RA: $18 \mathrm{~h} 40 \mathrm{~m} 37.61 \mathrm{~s}$ and DEC: $-05^{\circ} 43^{\prime} 17.99^{\prime \prime}$, which is $4.31^{\prime}$ away from the hard X-ray source (Pandey et al. 2006b). The NVSS image of the field also shows a point source of $165 \mathrm{mJy}$ coincident with the GMRT position. There are radio, optical and IR sources very close in position with respect to each other, and any of these could be related to the INTEGRAL source. The ASCA source lies further away and may be unassociated, nevertheless considering the large uncertainty in the X-ray position it is still possible that the ASCA source is associated with the INTEGRAL source. The radio spectrum of the GMRT source with the non-simultaneous VLA data suggest an absorption at low frequencies as seen in the case of other microquasars.

At VLBI scales the source is resolved out. There was no detection and the noise recorded in the map was 153 microJy/beam at $5 \mathrm{GHz}$. The source is positively detected at the level of $\sim 210$ mJy in the WSRT data and already shows resolved structure on the longest WSRT baselines. The noise recorded in the WSRT map was $2.43 \mathrm{mJy} /$ beam. Thus e-EVN observations has helped us to confirm that the possible radio counterpart for IGR J1804-0539 is not a jet source at milli arcsec resolution, and hence likely not a microquasar. Though IGR J18406-0539 is considered to be a $\mathrm{HMXB}$, there is no associated radio source found.

\section{Conclusion}

Microquasars allow us to study the relativistic jets and their interaction with the ISM. The connection between accretion disk instabilities and the formation of jets constrain the origin and evolution of black holes. INTEGRAL allows the discovery and re-discovery of highly absorbed sources in the Galactic plane, some of which (e.g. AX J1639.04642) are microquasar candidates. There is a lot of work in progress conducted by several groups to unveil the nature of the already known sources, and many more sources are discovered by INTEGRAL.

This work presents radio observations on 2 possible microquasar candidates discovered by the INTEGRAL satellite. Possible radio counterparts for two northern candidates, IGR J17303-0601 and IGR J18406-0539 were observed by the EVN to map their compactness. Our observations confirm that, IGR J17303-0601 has no radio emission detected in the ROSAT error circle and IGR J18406-0539 is resolved out at milliarcseccond scale.

The NVSS radio source near IGR J17303-0601 shows interesting extended morphology, but it is likely a background object. From these observations we conclude that the NVSS radio counterparts are not associated with IGR J18406-0539 and IGR J17303-0601. These e-EVN observations showed that the INTEGRAL sources are unlikely to be related to microquasars.

\section{Acknowledgements}

MP acknowledges the support of Prof. P. O. Laggage during her visit to CEA.

This work has benefited from research funding from the European Community's sixth Framework Programme under RadioNet R113CT 2003 5058187. e-VLBI developments in Europe are sup- 
ported by the EC DG-INFSO funded Communication Network Developments project 'EXPReS', Contract No. 02662 (http://www.expres-eu.org/). The European VLBI Network (http://www.evlbi.org/) is a joint facility of European, Chinese, South African and other radio astronomy institutes funded by their national research councils.

\section{References}

[1] Bamba A., Ueno M., Koyama K. and Yamauchi S., 2003, ApJ, 589, 253-260

[2] E.J. Barlow, C. Knigge, A.J. Bird., et al.,MNRAS, 372, 224

[3] Belanger G., Goldwurm A., Goldoni P., et al., 2004, ApJ, L163, 601

[4] Cutri R.M., Skrutskie M.F., Van Dyk S., et al., 2003, 2MASS All-Sky Catalog of Point Sources

[5] Fender, R.; Corbel, S., Tzioumis, T.; et al.; 1999, ApJ, 519L, 165

[6] Gansicke, B. T., Marsh, T. R., Edge, A., et al., 2005, ATEL, 463

[7] Masetti N., Palazzi E., Bassani L., et al., 2004, A\&A, 426L, 41

[8] Masetti N., Morelli, L.; Palazzi, E.; Galaz, G., et al. 2006; A\&A, 459, 21

[9] Mirabel F., Gerard E., Rodríguez L. 1994, IAU Circ., 5958

[10] Monet D., Bird A., Canzian B., et al., 1998, USNO-A2.0 Catalogue of Astrometric Standards

[11] Monet, D., Levine, S., Canzian, B., et al., 2003, AJ, 125, 984

[12] Pandey, M.; Rao, A. P.; Manchanda, R.; et al.,; 2006a, A\&A, 453, 83

[13] Pandey, M.; Manchanda, R. K.; Rao, A. P.; et al.,; 2006b, A\&A, 447, 525

[14] Paradijs V., \& McClintock J. 1995, in X-ray Binaries, ed. W. H. G. Lewin, J. van Paradijs, \& E. P. J. van den Heuvel, Cambridge Univ. Press, 58

[15] Stephen J., Bassini L., Molina M., et al., 2005, A\&A, 432, L49

[16] Voges W., Aschenbach B., Boller T., et al., 1999, A\&A, 349, 389

[17] Walter R., Bodaghee A., et al., 2004, ATEL 229 